\title{
Dual formulations of vortex strings in a supersymmetric Abelian Higgs model
}

\author{
Muneto Nitta ${ }^{*}$ and Ryo Yokokura ${ }^{\dagger}$ \\ Department of Physics \& Research and Education Center for Natural Sciences, Keio University, \\ Hiyoshi 4-1-1, Yokohama, Kanagawa 223-8521, Japan
}

(Received 23 February 2019; published 16 September 2019)

\begin{abstract}
We discuss dual formulations of vortex strings (magnetic flux tubes) in the four-dimensional $\mathcal{N}=1$ supersymmetric Abelian Higgs model with the Fayet-Iliopoulos term in the superspace formalism. The Lagrangian of the model is dualized into a Lagrangian of the $B F$ type described by a chiral spinor gauge superfield including a 2-form gauge field. The dual Lagrangian is further dualized into a Lagrangian given by a chiral spinor superfield including a massive 2 -form field. In both of the dual formulations, we obtain a superfield into which the vortex strings and their superpartners are embedded. We show the dual Lagrangians in terms of a superspace and a component formalism. In these dual Lagrangians, we explicitly show that the vortex strings of the original model are described by a string current electrically coupled with the 2-form gauge field or the massive 2-form field.
\end{abstract}

DOI: 10.1103/PhysRevD.100.065007

\section{INTRODUCTION}

Understanding phases of gauge theories is one of the important issues in quantum field theories. In the gauge theories, there is the so-called Higgs phase in which a gauge field becomes massive. One of the simplest renormalizable theories describing the Higgs phase may be the Abelian Higgs model, in which a $U(1)$ gauge field is coupled with a complex scalar field charged under the $U(1)$ symmetry. In the Higgs phase of the Abelian Higgs model, the $U(1)$ gauge field eats a phase part of the complex scalar field and becomes massive. Furthermore, there can exist extended objects of spatial dimension 1 as solutions of the equation of motion (EOM) in the Higgs phase. The extended objects are called Abrikosov-Nielsen-Olesen (ANO) vortex strings [1,2]. The ANO vortex strings are magnetic flux tubes which have topological charges, and they can be regarded as topological solitons. Such vortex strings arise in many contexts such as type-II superconductors [1] in condensed matter physics as well as cosmic strings [3-5] in cosmology (see, e.g., Refs. [6,7] as a review).

While the ANO vortex strings are introduced as solutions to the EOM, they can be seen as charged objects associated with gauge fields by using dual transformations. For the

\footnotetext{
"nitta@phys-h.keio.ac.jp

ryokokur@keio.jp
}

Published by the American Physical Society under the terms of the Creative Commons Attribution 4.0 International license. Further distribution of this work must maintain attribution to the author(s) and the published article's title, journal citation, and DOI. Funded by SCOAP ${ }^{3}$.
Abelian Higgs model, there are at least two dual formulations. One is to dualize the phase of the scalar field to a 2-form gauge field [8-11]. In this dual formulation, the original 1-form gauge field and the dualized 2-form gauge field are massive by the topological coupling (the so-called $B F$ coupling) between them $[12,13]$. In this dual formulation, the ANO vortex strings are described by a conserved string current which is electrically coupled with the 2-form gauge field $[14,15]$. Another is to dualize the massive 1-form field to a massive 2-form field [16]. The 1-form gauge field becomes massive after eating the phase of the complex scalar field. The dual 2-form field can be regarded as a 2-form gauge field eating the 1-form gauge field by a Stückelberg coupling. In this dual formulation, too, the ANO vortex strings are dualized to a string current electrically coupled with the massive 2 -form field $[17,18]$. The dual transformation was applied to a finite-temperature phase transition of the Abelian Higgs model [19].

In the Abelian Higgs model, the positions of the ANO vortex strings are characterized by zero points of the complex scalar field, and the dual string current is described by the singularities due to a multivalued part of the phase of the complex scalar field around the zero points (see, e.g., Ref. [20]). The dual formulation with ANO vortex strings can be obtained by splitting the complex scalar field into the regular part and the singular part. The phase in the regular part of the complex scalar field can be dualized into the 2 -form gauge field. On the other hand, the phase of the singular part, which is the multivalued function, is dualized to the string current.

There are some virtues of the dual transformations. One virtue of the dual formulations is that the topological charge 
of the ANO vortex strings can be simply understood as the conserved charge associated with the gauge symmetry for the 2-form field [21]. Another virtue is that the ANO vortex strings become fundamental degrees of freedom in contrast to the original theory.

In the literature, there are some generalizations of the duality of ANO vortex strings in the Abelian Higgs model. One is the case of global strings in the Goldstone model, that is, a $U(1)$ Higgs model without a gauge interaction. In this case, a Nambu-Goldstone boson associated with the spontaneously broken global $U(1)$ symmetry is dualized to a massless 2-form field, and global strings are electrically coupled to the 2 -form field $[15,22]$. These strings are axion strings in cosmology and superfluid vortices in superfluids in condensed matter physics. Another generalization is the case of non-Abelian gauge theories. An $S U(2)$ gauge theory coupled with one complex (two real) adjoint Higgs fields is known to admit $\mathbb{Z}_{2}$ strings [2]. A nonAbelian duality in this case was obtained in Ref. [23], in which the dual Lagrangian is described by a non-Abelian 2 -form field [23,24] coupled with $\mathbb{Z}_{2}$ strings. Another case is an $S U(3)$ gauge theory coupled with three by three complex Higgs fields in the fundamental representation, relevant for QCD at high density and low temperature. This theory admits a non-Abelian vortex (color flux tubes) [25-27], accompanied with non-Abelian $\mathbb{C} P^{2}$ moduli [28], and a non-Abelian duality of non-Abelian vortices in this theory was obtained in Refs. [27,29].

In general, there are attractive and repulsive forces among the ANO vortex strings intermediated by Higgs and gauge fields, respectively. For type-II (I) superconductors, the gauge field is lighter (heavier) than the Higgs field; thereby, repulsion (attraction) is dominant. The multiple vortex strings become stable if the two forces are balanced at the critical coupling between type-I and type-II superconductors. Such a state is called a Bogomol'nyi-PrasadSommerfield (BPS) state $[30,31]$. In the BPS state, the total mass of the ANO vortex strings is proportional to the total topological charge (see, e.g., Ref. [32]).

Supersymmetry (SUSY) gives us nonperturbative aspects of BPS states [33]. The BPS states preserve half of the SUSY charges if the theories are embedded into SUSY theories. Since the BPS states are protected by SUSY, the BPS states are stable against quantum corrections [34]. The SUSY Abelian Higgs model [35] can be constructed by using a vector gauge superfield (so-called 1-form prepotential) with a Fayet-Iliopoulos (FI) term [36] and chiral superfields charged under the $U(1)$ gauge symmetry. In particular, the ANO vortex strings can be constructed by using a D-term potential [37-42].

The dual formulations of the SUSY Abelian Higgs model are possible. In SUSY theories, the duality between the scalar field and the 2-form field can be extended into the duality between a chiral superfield and a chiral spinor gauge superfield [43-45], which we will call "2-form prepotential" [46]. This is because the 2-form gauge field can be embedded into the chiral spinor gauge superfield. Furthermore, the duality between a massive 1-form field and a massive 2-form field can also be understood as the duality between a real superfield and a chiral spinor superfield [47-50]. Such dual transformations were extended to supergravity (SUGRA) [49] and extended SUSY theories [49]. However, the superfield descriptions of the dual formulations of the ANO vortex strings in the SUSY context have not been understood so far. The abovementioned dual formulations in SUSY theories only describe the regular part without singularities. To understand nonperturbative aspects of the ANO vortex strings in SUSY theories, it is plausible to dualize the SUSY Abelian Higgs model including the ANO vortex strings in a manifestly SUSY way.

In this paper, we show the dual formulations of the fourdimensional (4D) $\mathcal{N}=1$ SUSY Abelian Higgs model including the ANO vortex strings. We use the superspace formalism in order to give the manifestly SUSY theories. There are at least two ways to dualize the Lagrangian of the Abelian Higgs model as mentioned above. We discuss both of the dual transformations to the theories with a 2-form gauge field and a massive 2-form field. In both of the dual formulations, we show the dual transformations of the ANO vortex strings in terms of superfields. As in the bosonic Abelian Higgs model, we split a chiral superfield describing the complex scalar field into the regular part and the singular part. For the regular part, there are no zero points of the complex scalar field. Therefore, the regular part of the chiral superfield can be dualized into the 2-form prepotential. For the singular part, the duality transformations give us the electrical coupling of the 2-form prepotential with a superfield given by the singular part. We show that the superfield given by the singular part has the string current as well as superpartners of the string current by the component expression of the dual Lagrangian. We can further dualize the 1-form prepotential. In this dual transformation, the Lagrangian can be written in terms of a massive chiral spinor superfield and the superfield into which the string current is embedded.

This paper is organized as follows. In Sec. II, we review the dual transformations of ANO vortex strings in the Abelian Higgs model without SUSY. In Sec. III, we show the duality transformations of ANO vortex strings in the SUSY Abelian Higgs model. We summarize this paper in Sec. IV. We use the notation and convention of the textbook in Ref. [51].

\section{DUAL TRANSFORMATIONS OF VORTEX STRINGS IN ABELIAN HIGGS MODEL}

In this section, we review two dual transformations of the ANO vortex strings of the bosonic Abelian Higgs model $[15,17]$ at a classical level. One is the transformation to the system described by a 1-form gauge field and a 2-form 
gauge field, in which the ANO vortex strings are electrically coupled with the 2-form gauge field. The other is the transformation to the system described by a massive 2-form field, which is also coupled with the ANO vortex strings.

\section{A. Abelian Higgs model}

Here, we introduce the Lagrangian of the Abelian Higgs model. The Lagrangian is given by

$\mathcal{L}_{\mathrm{AH}}=-\left|\partial_{m} \phi-\frac{e}{2} i A_{m} \phi\right|^{2}-\frac{1}{4} F^{m n} F_{m n}-\frac{1}{8}\left(e|\phi|^{2}-\xi\right)^{2}$.

Here, $A_{m}(m=0,1,2,3)$ is a $U(1)$ gauge field, $F_{m n}=$ $\partial_{m} A_{n}-\partial_{n} A_{m}$ is the field strength of the gauge field, $\phi$ is a complex scalar field with the $U(1)$ charge $e / 2, e$ is a positive coupling constant of the $U(1)$ gauge field, and $\xi$ is a positive parameter of mass dimension 2 . Note that the parameters are normalized so that the model can be embedded into SUSY theories. The vacuum of the model is given by the minimum of the potential where $|\phi|$ develops nonzero vacuum expectation value

$$
|\phi|^{2}=\frac{\xi}{e}
$$

Therefore, the $U(1)$ symmetry is spontaneously broken in this vacuum. The vacuum is in the Higgs phase since the gauge field becomes massive by eating the phase of the scalar field.

\section{B. Dual 2-form gauge theory with vortex strings}

The Higgs phase admits spatial dimension-1 (codimension-2) objects, since the first homotopy group of the vacuum manifold is nontrivial: $\pi_{1}(U(1))=\mathbb{Z}$. The extended objects are so-called ANO vortex strings. The positions of the ANO vortex strings are characterized by the zero points of $\phi$, where the $U(1)$ symmetry is recovered.

In the Lagrangian, the ANO vortex strings are expressed by using the multivalued part of the phase of the complex scalar field (see, e.g., Ref. [20]). We split the complex scalar field as follows:

$$
\phi=\frac{1}{\sqrt{2}} \rho e^{i\left(\varphi+\varphi_{0}\right)} .
$$

Here, $\rho$ and $\varphi$ are real single-valued scalar fields, and $\varphi_{0}$ is a real multivalued scalar field. In general, the phase can be multivalued since $\varphi_{0} \rightarrow \varphi_{0}+2 \pi$ does not change $\phi$. The Lagrangian in Eq. (2.1) can be rewritten as

$$
\begin{aligned}
\mathcal{L}_{\mathrm{AH}}= & -\left|\partial_{m} \phi-i \frac{e}{2} A_{m} \phi\right|^{2}-\frac{1}{4} F^{m n} F_{m n}+\cdots \\
= & -\frac{1}{2}\left|\partial_{m} \rho+i\left(\partial_{m} \varphi+\partial_{m} \varphi_{0}\right) \rho-i \frac{e}{2} A_{m} \rho\right|^{2} \\
& -\frac{1}{4} F^{m n} F_{m n}+\cdots \\
= & -\frac{1}{2}\left(\partial_{m} \rho\right)^{2}-\frac{1}{2} \rho^{2}\left(\partial_{m} \varphi+\partial_{m} \varphi_{0}-\frac{e}{2} A_{m}\right)^{2} \\
& -\frac{1}{4} F^{m n} F_{m n}+\cdots,
\end{aligned}
$$

where the ellipsis refers to the terms which are irrelevant to the dual formulations.

We dualize the scalar field $\varphi$ to a 2-form gauge field as follows. We introduce the first-order Lagrangian, which is classically equivalent to the Lagrangian in Eq. (2.4),

$$
\begin{aligned}
\mathcal{L}_{B, 1 \mathrm{st}}= & -\frac{1}{2} \rho^{2}\left(C_{m}+\partial_{m} \varphi_{0}-\frac{e}{2} A_{m}\right)^{2} \\
& +\frac{1}{2 !} \epsilon^{m n p q} B_{m n} \partial_{p} C_{q}-\frac{1}{4} F^{m n} F_{m n},
\end{aligned}
$$

where we have omitted the terms which are irrelevant to the following discussions. Here, $C_{m}$ is a 1-form gauge field without singularities, and $B_{m n}$ is a 2-form gauge field. The gauge field $B_{m n}$ is transformed as $B_{m n} \rightarrow B_{m n}+\partial_{m} \lambda_{n}-\partial_{n} \lambda_{m}$, where $\lambda_{m}$ is a 1-form gauge parameter. The gauge field $C_{m}$ is transformed under the gauge transformation of $A_{m} \rightarrow A_{m}+\partial_{m} u$ as $C_{m} \rightarrow C_{m}+$ $\frac{e}{2} \partial_{m} u$, where $u$ is a gauge parameter. The equivalence between the Lagrangian and the one in Eq. (2.4) can be seen by solving the EOM for $B_{m n}$, which gives us $C_{m}=\partial_{m} \varphi$. The dual formulation can be obtained by using the EOM for $C_{m}$ and by eliminating the field. The EOM for the $C_{m}$ gives us

$$
C_{m}=\frac{1}{\rho^{2}}(* H)_{m}-\partial_{m} \varphi_{0}+\frac{e}{2} A_{m},
$$

where we have defined

$$
H_{m n p}:=\partial_{m} B_{n p}+\partial_{n} B_{p m}+\partial_{p} B_{m n}
$$

and

$$
(* H)^{m}:=\frac{1}{3 !} \epsilon^{m n p q} H_{n p q} .
$$

Therefore, the first-order Lagrangian in Eq. (2.5) becomes 


$$
\begin{aligned}
\mathcal{L}_{B}= & \frac{1}{2 \rho^{2}}(* H)^{m}(* H)_{m}-\frac{1}{4} F^{m n} F_{m n}+\frac{e}{2} \cdot \frac{1}{2 ! \cdot 2 !} \epsilon^{m n p q} B_{m n} F_{p q} \\
& -\frac{1}{2 !} \epsilon^{m n p q} B_{m n} \partial_{p} \partial_{q} \varphi_{0} .
\end{aligned}
$$

The Lagrangian describes a system with 1-form and 2-form gauge field with the topological coupling $\epsilon^{m n p q} B_{m n} F_{p q}$. The 2-form gauge field is electrically coupled with the string current $\epsilon^{m n p q} \partial_{p} \partial_{q} \varphi_{0}$. Naively, $\epsilon^{m n p q} \partial_{p} \partial_{q} \varphi_{0}$ seems to be identically zero. However, since $\varphi_{0}$ is a multivalued function, $\epsilon^{m n p q} \partial_{p} \partial_{q} \varphi_{0}$ is nonzero where $|\phi|$ becomes zero. This can be seen as follows. Since $\varphi_{0}$ is a part of the phase of the complex scalar field, $\epsilon^{m n p q} \partial_{p} \partial_{q} \varphi_{0}$ can be rewritten as follows:

$$
\epsilon^{m n p q} \partial_{p} \partial_{q} \varphi_{0}=\frac{1}{2 i} \epsilon^{m n p q} \partial_{p} \partial_{q} \log (\phi / \bar{\phi}) .
$$

Here, we have included the regular part $\varphi$ since $\epsilon^{m n p q} \partial_{p} \partial_{q} \varphi=0$. The right-hand side of Eq. (2.10) gives rise to a delta function:

$$
\begin{aligned}
\frac{1}{2 i} \epsilon^{m n p q} \partial_{p} \partial_{q} \log (\phi / \bar{\phi}) & =\frac{1}{2 i} \epsilon^{m n p q} \partial_{p}\left(\frac{1}{\phi} \partial_{q} \phi-\frac{1}{\bar{\phi}} \partial_{q} \bar{\phi}\right) \\
& =-2 \pi i \epsilon^{m n p q} \delta^{2}(\phi, \bar{\phi}) \partial_{p} \bar{\phi} \partial_{q} \phi .
\end{aligned}
$$

Here, we have used a property of a two-dimensional delta function,

$$
\frac{\partial}{\partial \bar{\phi}} \frac{1}{\phi}=\frac{\partial}{\partial \phi} \frac{1}{\bar{\phi}}=2 \pi \delta^{2}(\phi, \bar{\phi}),
$$

where the delta function is defined by

$$
\delta^{2}(\phi, \bar{\phi}):=\frac{1}{2} \delta(\operatorname{Re} \phi) \delta(\operatorname{Im} \phi) .
$$

Thus, $\epsilon^{m n p q} \partial_{p} \partial_{q} \varphi_{0}$ has singularities of the delta function where $|\phi|$ is zero. The string current is a conserved current,

$$
\partial_{n} \epsilon^{m n p q} \partial_{p} \partial_{q} \varphi_{0}=0
$$

because $\epsilon^{m n p q} \partial_{n} \phi \partial_{p} \phi \partial_{q} \bar{\phi}=\epsilon^{m n p q} \partial_{n} \phi \partial_{p} \bar{\phi} \partial_{q} \bar{\phi}=0$.

\section{Dual massive 2-form theory with vortex strings}

We have dualized the Lagrangian in Eq. (2.4) into the one with the 2-form gauge field. We can also dualize the Lagrangian into a system with a massive 2 -form field. We introduce the following first-order Lagrangian, which is classically equivalent to the Lagrangian in Eq. (2.9):

$$
\begin{aligned}
\mathcal{L}_{B^{\prime}, 1 \mathrm{st}}= & \frac{1}{2 \rho^{2}}(* H)^{m}(* H)_{m}-\frac{1}{2 !} \epsilon^{m n p q} B_{m n} \partial_{p} \partial_{q} \varphi_{0} \\
& +\frac{e}{2} \cdot \frac{1}{3 !} \epsilon^{m n p q} A_{m} H_{n p q}-\frac{1}{4} F^{\prime m n} F_{m n}^{\prime} \\
& +\frac{1}{2 ! \cdot 2 !} \epsilon^{m n p q} B_{m n}^{\prime}\left(\partial_{p} A_{q}-\partial_{q} A_{p}-F_{p q}^{\prime}\right) .
\end{aligned}
$$

Here, $B_{m n}^{\prime}$ is a 2-form field as a Lagrange multiplier, and $F_{m n}^{\prime}$ is a 2-form field which is independent of the original 1-form gauge field $A_{m}$. The EOM for the Lagrange multiplier gives us the relation $F_{m n}^{\prime}=\partial_{m} A_{n}-\partial_{n} A_{m}$, and we go back to the original Lagrangian in Eq. (2.9). Instead, the EOM for $A_{m}$ gives us

$$
\frac{1}{3 !} \epsilon^{m n p q} H_{n p q}=-\frac{2}{e} \cdot \frac{1}{2 !} \epsilon^{m n p q} \partial_{n} B_{p q}^{\prime} .
$$

Furthermore, the EOM for $F_{m n}^{\prime}$ leads to

$$
F_{m n}^{\prime}=-\frac{1}{2 !} \epsilon_{m n p q} B^{\prime p q} .
$$

Substituting Eqs. (2.16) and (2.16) into the Lagrangian in Eq. (2.15), we obtain

$$
\begin{aligned}
\mathcal{L}_{B^{\prime}}= & \frac{2}{e^{2} \rho^{2}}\left(* H^{\prime}\right)^{m}\left(* H^{\prime}\right)_{m}-\frac{1}{4} B^{\prime m n} B_{m n}^{\prime} \\
& -\frac{2}{e} \cdot \frac{1}{2 !} \epsilon^{m n p q} B_{m n}^{\prime} \partial_{p} \partial_{q} \varphi_{0}
\end{aligned}
$$

up to total derivatives. Here, we have defined

$$
\left(* H^{\prime}\right)^{m}=\frac{1}{2 !} \epsilon^{m n p q} \partial_{n} B_{p q}^{\prime} .
$$

The second term of the Lagrangian in Eq. (2.18) is the mass term for the 2-from $B_{m n}^{\prime}$. Therefore, the Lagrangian in Eq. (2.18) describes a system of a massive 2-form field. The ANO vortex strings are coupled with the massive 2-form.

\section{DUAL TRANSFORMATIONS OF VORTEX STRINGS IN SUSY ABELIAN HIGGS MODEL}

In this section, we discuss the dual transformations of ANO vortex strings of the SUSY Abelian Higgs model. In SUSY theories, the Higgs potential can be obtained by F-term or a D-term potentials [39]. For the former case, the SUSY is completely broken in the core of the vortex strings. For the latter case, half of SUSY can be preserved in the core of the ANO vortex strings, and the ANO vortex strings can be BPS states [39-41]. We thus discuss the latter option in this paper.

We use the superspace formalism in order to obtain the manifestly SUSY theories. The superspace is spanned by the coordinates $\left(x^{m}, \theta^{\alpha}, \bar{\theta}_{\dot{\alpha}}\right)$, where $\left(x^{m}\right)(m=0,1,2,3)$ are coordinates of the Minkowski spacetime and $\left(\theta^{\alpha}, \bar{\theta}_{\dot{\alpha}}\right)$ are coordinates spanned by the Grassmann numbers. The indices beginning with $m, n, \ldots$ are vector indices. 
The indices beginning with $\alpha, \beta, \ldots \dot{\alpha}, \dot{\beta}, \ldots$ are spinor indices with $\alpha=1,2$ and $\dot{\alpha}=\dot{1}, \dot{2}$.

\section{A. SUSY Abelian Higgs model}

We introduce a Lagrangian of the SUSY Abelian Higgs model. We begin with the following Lagrangian:

$$
\begin{aligned}
\mathcal{L}_{\mathrm{AH}, \mathrm{SUSY}}= & \frac{1}{2} \int d^{4} \theta\left(\bar{\Phi} e^{\mathrm{eV}} \Phi+\overline{\tilde{\Phi}} e^{-e V} \tilde{\Phi}-\xi V\right) \\
& +\frac{1}{4} \int d^{2} \theta W^{\alpha} W_{\alpha}+\text { H.c. }
\end{aligned}
$$

Here, $V$ is a vector superfield in which a $U(1)$ vector gauge field $A_{m}$ is embedded, $W_{\alpha}=-\frac{1}{4} \bar{D}^{2} D_{\alpha} V$ is a gaugino superfield given by the vector superfield, $e$ is a positive coupling constant of the $U(1)$ gauge symmetry, and $\xi$ is a FI parameter [36]. Superfields $\Phi$ and $\tilde{\Phi}$ are chiral superfields with $U(1)$ charge $+e / 2$ and $-e / 2$, respectively. The chiral superfields are transformed by the $U(1)$ gauge transformation as $\Phi \rightarrow \Phi e^{e \Lambda}$ and $\tilde{\Phi} \rightarrow \tilde{\Phi} e^{-e \Lambda}$ when $V$ is transformed as $V \rightarrow V-\Lambda-\bar{\Lambda}$. Here, $\Lambda$ is a chiral superfield parameter. The bosonic part of the component Lagrangian is

$$
\begin{aligned}
\mathcal{L}_{\mathrm{AH}, \text { SUSY,boson }}= & -\left|\partial_{m} \phi-i \frac{e}{2} A_{m} \phi\right|^{2}-\left|\partial_{m} \tilde{\phi}+i \frac{e}{2} A_{m} \tilde{\phi}\right|^{2} \\
& -\frac{1}{4} F^{m n} F_{m n}+\frac{1}{2} \boldsymbol{D}\left(e|\phi|^{2}-e|\tilde{\phi}|^{2}-\xi\right) \\
& +F \bar{F}+\tilde{F} \overline{\tilde{F}}+\frac{1}{2} \boldsymbol{D}^{2} .
\end{aligned}
$$

Here, we have omitted fermions which are not needed for the following discussion in this section. In the Lagrangian Eq. (3.2), we have used the Wess-Zumino (WZ) gauge: $V\left|=D_{\alpha} V\right|=\bar{D}_{\dot{\alpha}} V\left|=D^{2} V\right|=\bar{D}^{2} V \mid=0$. Here, the vertical bar $\mid$ represents $\theta=\bar{\theta}=0$ projection of the superfields, and $D_{\alpha}$ and $\bar{D}_{\dot{\alpha}}$ are SUSY-covariant spinor derivatives. The components of the chiral superfield $\Phi$ and the vector superfield $V$ are denoted as

$$
\begin{aligned}
& \phi=\Phi\left|, \quad \chi_{\alpha}=\frac{1}{\sqrt{2}} D_{\alpha} \Phi\right|, \quad F=-\frac{1}{4} D^{2} \Phi \mid, \\
& \tilde{\phi}=\tilde{\Phi}\left|, \quad \tilde{\chi}_{\alpha}=\frac{1}{\sqrt{2}} D_{\alpha} \tilde{\Phi}\right|, \quad \tilde{F}=-\frac{1}{4} D^{2} \tilde{\Phi} \mid, \\
& A_{\alpha \dot{\alpha}}=\frac{1}{2}\left[D_{\alpha}, \bar{D}_{\dot{\alpha}}\right] V \mid, \\
& F_{m n}=\partial_{m} A_{n}-\partial_{n} A_{m}=\frac{1}{2 i}\left(\left(\sigma_{m n}\right)_{\alpha}^{\beta} D^{\alpha} W_{\beta}-\left(\bar{\sigma}_{m n}\right)^{\dot{\alpha}} \bar{D}_{\dot{\beta}} \bar{D}_{\dot{\alpha}} \bar{W}^{\dot{\beta}}\right) \mid, \\
& \lambda_{\alpha}=i W_{\alpha}\left|, \quad \bar{\lambda}^{\dot{\alpha}}=-i \bar{W}^{\dot{\alpha}}\right|, \quad \boldsymbol{D}=-\frac{1}{2} D^{\alpha} W_{\alpha}\left|=-\frac{1}{2} \bar{D}_{\dot{\alpha}} \bar{W}^{\dot{\alpha}}\right| .
\end{aligned}
$$

The quantities $\left(\sigma^{m}\right)_{\alpha \dot{\alpha}}$ and $\left(\bar{\sigma}^{m}\right)^{\dot{\alpha} \alpha}$ are four-dimensional Pauli matrices which satisfy $\left(\bar{\sigma}^{m}\right)^{\dot{\alpha} \alpha}=\left(\sigma^{m}\right)^{\alpha \dot{\alpha}}$. The quantity $A_{\alpha \dot{\alpha}}$ is defined by the Pauli matrices as $A_{\alpha \dot{\alpha}}=\left(\sigma^{m}\right)_{\alpha \dot{\alpha}} A_{m}$. The quantities $\left(\sigma^{m n}\right)_{\alpha}{ }^{\beta}$ and $\left(\bar{\sigma}^{m n}\right)_{\dot{\beta}}^{\dot{\alpha}}$ are self-dual and antiself dual tensors defined by

$$
\begin{aligned}
& \left(\sigma^{m n}\right)_{\alpha}^{\beta}=\frac{1}{4}\left(\left(\sigma^{m}\right)_{\alpha \dot{\gamma}}\left(\bar{\sigma}^{n}\right)^{\dot{\gamma} \beta}-\left(\sigma^{n}\right)_{\alpha \dot{\gamma}}\left(\bar{\sigma}^{m}\right)^{\dot{\gamma} \beta}\right), \\
& \left(\bar{\sigma}^{m n}\right)_{\dot{\beta}}^{\dot{\alpha}}=\frac{1}{4}\left(\left(\bar{\sigma}^{m}\right)^{\dot{\alpha} \gamma}\left(\sigma^{n}\right)_{\gamma \dot{\beta}}-\left(\bar{\sigma}^{n}\right)^{\dot{\gamma} \gamma}\left(\sigma^{m}\right)_{\gamma \dot{\beta}}\right),
\end{aligned}
$$

respectively.

In this model, the $U(1)$ symmetry is spontaneously broken if the FI parameter $\xi$ is nonzero. This can be seen by the on-shell potential $\mathcal{V}$,

$\mathcal{V}=-\frac{1}{2} \boldsymbol{D}\left(e|\phi|^{2}-e|\tilde{\phi}|^{2}-\xi\right)-F \bar{F}-\tilde{F} \overline{\tilde{F}}-\frac{1}{2} \boldsymbol{D}^{2}$.

To obtain the on-shell potential, we solve the EOM for the auxiliary fields $F$ and $\boldsymbol{D}$. The EOM for $F$ and $\tilde{F}$ are trivial, $F=\tilde{F}=0$, while the EOM for $\boldsymbol{D}$ is

$$
\boldsymbol{D}=-\frac{1}{2}\left(e|\phi|^{2}-e|\tilde{\phi}|^{2}-\xi\right) .
$$

Therefore, the on-shell potential $\mathcal{V}$ is

$$
\mathcal{V}=\frac{1}{8}\left(e|\phi|^{2}-e|\tilde{\phi}|^{2}-\xi\right)^{2} .
$$

The vacuum of the model is given by the minimum of the potential, which is described by the condition

$$
e|\phi|^{2}-e|\tilde{\phi}|^{2}=\xi .
$$

If the FI parameter is positive $\xi>0,|\phi|^{2}$ cannot be zero, while $|\tilde{\phi}|^{2}$ can be zero. Since $\phi$ develops the vacuum expectation value, the $U(1)$ symmetry is broken, and the vector field $A_{m}$ becomes massive by eating the phase of $\phi$. Note that SUSY is unbroken in this vacuum since the vacuum expectation value of the auxiliary field is $\boldsymbol{D}=0$ in this vacuum.

\section{B. Dual SUSY 2-form gauge theory with vortex strings}

We consider a dual formulation of the SUSY Abelian Higgs model. We use the superspace formalism in order to make SUSY manifest. In Sec. II B, we have reviewed the dual transformations of the bosonic Abelian Higgs model. As in the bosonic Abelian Higgs model, there are at least two ways to dualize the Lagrangian. One is to dualize the chiral superfield $\Phi$. In this case, the dual theory is described by a 2 -form gauge field $B_{m n}$ in addition to the original 1-form gauge field $A_{m}$. In the dual theory, the 2-form gauge field is topologically coupled with the 1-form gauge field. 
The other is to dualize the vector superfield $V$. In this case, the dual theory is described by a massive 2 -form field where the 1 -form gauge field is eaten by the 2 -form gauge field. In this subsection, we choose the former option. The ANO vortex strings are coupled with the 2 -form gauge field electrically.

\section{String current superfield}

We begin with the Lagrangian

$\mathcal{L}_{\mathrm{AH}, \mathrm{SUSY}}^{\prime}=\frac{1}{2} \int d^{4} \theta\left(\bar{\Phi} e^{\mathrm{eV}} \Phi-\xi V\right)+\frac{1}{4} \int d^{2} \theta W^{\alpha} W_{\alpha}+$ H.c.,

where we have omitted the terms which are irrelevant to the ANO vortex strings, since we are interested in the dual formulation of the ANO vortex strings. In the presence of the vortex strings, the Lagrangian has singular points in the field space of $\Phi$ where $\Phi=0$. To dualize the Lagrangian, we split $\Phi$ into the singular part and the regular part as follows:

$$
\Phi=\Phi_{0} \Phi_{1}, \quad \text { where } \Phi_{0}:=\frac{\Phi}{\Phi_{1}} .
$$

Here, $\Phi_{1}$ is a regular chiral superfield of mass dimension 1, which does not have a zero point. This regular part can be understood as a SUSY extension of $e^{i \varphi}$ in the bosonic model in Sec. II B. Since $\Phi_{1}$ is the regular chiral superfield, we can assign nonsingular gauge transformations for $\Phi_{1}$. We assume the same gauge transformation law of the chiral superfield $\Phi_{1}$ as $\Phi: \Phi_{1} \rightarrow \Phi_{1} e^{e \Lambda}$. Since $\Phi_{1}$ is not zero everywhere, there are no singular points for the gauge transformation. On the other hand, $\Phi_{0}$ has singular points where $\Phi_{0}$ is zero. Again, this singular part can be understood as a SUSY extension of $e^{i \varphi_{0}}$ in the bosonic model in Sec. II B. This zero point is originated from the zero point of the chiral superfield $\Phi$. Thanks to the splitting $\Phi=$ $\Phi_{0} \Phi_{1}$, we can discuss the regular and singular parts in manifestly gauge-covariant and -invariant ways, respectively.

We rewrite the Lagrangian in Eq. (3.2) by using $\Phi_{0}$ and $\Phi_{1}$ as follows:

$$
\begin{aligned}
\mathcal{L}_{\mathrm{AH}, \mathrm{SUSY}}^{\prime}= & \frac{1}{2} \int d^{4} \theta\left|\Phi_{0}\right|^{2}\left|\Phi_{1}\right|^{2} e^{\mathrm{eV}}+\frac{1}{4} \int d^{2} \theta W^{\alpha} W_{\alpha} \\
& -\frac{1}{2} \xi \int d^{4} \theta V+\text { H.c. }
\end{aligned}
$$

Now, we dualize $\left|\Phi_{1}\right|^{2}$ by the following the first-order Lagrangian:

$$
\begin{aligned}
\mathcal{L}_{B, \mathrm{SUSY}, 1 \mathrm{st}}^{\prime}= & \frac{1}{2} \int d^{4} \theta\left|\Phi_{0}\right|^{2} M^{2} e^{U+\mathrm{eV}}+\frac{1}{4} \int d^{2} \theta W^{\alpha} W_{\alpha} \\
& -\frac{1}{2} \xi \int d^{4} \theta V-\frac{1}{4 \cdot 2 i} \int d^{2} \theta \Sigma^{\alpha} \bar{D}^{2} D_{\alpha} U+\text { H.c. }
\end{aligned}
$$

Here, $U$ is a real superfield of which the gauge transformation law is $U \rightarrow U+e(\Lambda+\bar{\Lambda})$ under $V \rightarrow V-\Lambda-\bar{\Lambda}$. The superfield $\Sigma_{\alpha}$ is a chiral superfield, and $M$ is a parameter of mass dimension 1 . Since the original chiral superfield $\Phi_{1}$ is regular, we can safely assume that $U$ is also a regular function in the sense that $e^{U}$ does not have zero points. The Lagrangian is invariant under the gauge transformation of $\Sigma_{\alpha}$,

$$
\delta_{2} \Sigma_{\alpha}=-\frac{1}{4} \bar{D}^{2} D_{\alpha} \Theta
$$

where $\delta_{2}$ refers to an infinitesimal gauge transformation of $\Sigma_{\alpha}$ and $\Theta$ is a real superfield parameter. Since the chiral spinor superfield with the gauge transformation in Eq. (3.15) includes the 2-form gauge field $B_{m n}$ as a component field (see, e.g., Ref. [44]), we call $\Sigma_{\alpha}$ a "2form prepotential" following Ref. [46].

We can go back to the original Lagrangian in Eq. (3.2) by eliminating $\Sigma_{\alpha}$ by its EOM. The EOM for $\Sigma_{\alpha}$ and its Hermitian conjugate,

$$
\bar{D}^{2} D_{\alpha} U=D^{2} \bar{D}_{\dot{\alpha}} U=0,
$$

give us the solution

$$
U=\Phi^{\prime}+\bar{\Phi}^{\prime},
$$

where $\Phi^{\prime}$ is a single-valued chiral superfield since $e^{U}$ is a nonzero superfield. If we define $\Phi_{1}=e^{\Phi^{\prime}}$, we obtain the original Lagrangian.

The dual formulation can be obtained by eliminating the real superfield $U$ instead of eliminating $\Sigma_{\alpha}$. The EOM for $U$ is

$$
0=\left|\Phi_{0}\right|^{2} M^{2} e^{U+\mathrm{eV}}-L,
$$

where $L$ is a real superfield defined by

$$
L=\frac{1}{2 i}\left(D^{\alpha} \Sigma_{\alpha}-\bar{D}_{\dot{\alpha}} \bar{\Sigma}^{\dot{\alpha}}\right) .
$$

Note that the real superfield $L$ is a linear superfield since $D^{2} L=\bar{D}^{2} L=0$.

By using the real linear superfield $L, U$ can be solved as

$$
U=\log \frac{L}{\left|\Phi_{0}\right|^{2} M^{2} e^{\mathrm{eV}}} .
$$


Substituting the solution into the first-order Lagrangian in Eq. (3.14), we reach the following dual Lagrangian:

$$
\begin{aligned}
\mathcal{L}_{B, \mathrm{SUSY}}^{\prime}= & -\frac{1}{2} \int d^{4} \theta L \log \left(\frac{L}{M^{2}}\right)+\frac{1}{4} \int d^{2} \theta W^{\alpha} W_{\alpha} \\
& -\frac{1}{2} \int d^{4} \theta \xi V-\frac{e}{2 i} \int d^{2} \theta \Sigma^{\alpha} W_{\alpha} \\
& -\frac{1}{2 i} \int d^{2} \theta \Sigma^{\alpha} J_{\alpha}+\text { H.c. }
\end{aligned}
$$

Here, we have defined the chiral superfield $J_{\alpha}$ as

$$
J_{\alpha}:=-\frac{1}{4} \bar{D}^{2} D_{\alpha} \log \left|\Phi_{0}\right|^{2}
$$

and we call the superfield $J_{\alpha}$ a "string current superfield" for later convenience. The terms $\frac{1}{2 i} \int d^{2} \theta \Sigma^{\alpha} J_{\alpha}+$ H.c. are invariant under the gauge transformation in Eq. (3.15) because $J_{\alpha}$ satisfies

$$
D^{\alpha} J_{\alpha}=\bar{D}_{\dot{\alpha}} \bar{J}^{\dot{\alpha}} .
$$

Naively, $\quad J_{\alpha}=0 \quad$ since $\log \left|\Phi_{0}\right|^{2}=\log \Phi_{0}+\log \bar{\Phi}_{0}$. However, since $\Phi_{0}$ can have zero points, $\bar{D}^{2} D_{\alpha} \log \left|\Phi_{0}\right|^{2}$ contains a singularity of a delta function. We will discuss the singularity more precisely.

\section{Component expression of dual formulation}

In the Lagrangian in Eq. (3.21), there is a coupling between the 2-form gauge field and the string current and its SUSY completion. The coupling and its SUSY completion are given by the last term. To see the coupling, we express the dual Lagrangian $\mathcal{L}_{B, \mathrm{SUSY}}^{\prime}$ in terms of the component fields. The component expression is

$$
\begin{aligned}
\mathcal{L}_{B, \mathrm{SUSY}}^{\prime}= & -\frac{1}{2 \sqrt{2} \sigma}\left(\left(\partial^{m} \sigma\right)\left(\partial_{m} \sigma\right)-(* H)^{m}(* H)_{m}\right)-\frac{i}{2 \sqrt{2} \sigma}\left(\bar{\psi}_{\dot{\alpha}}\left(\bar{\sigma}^{m}\right)^{\dot{\alpha} \alpha} \partial_{m} \psi_{\alpha}+\psi^{\alpha}\left(\sigma^{m}\right)_{\alpha \dot{\alpha}} \partial_{m} \bar{\psi}^{\dot{\alpha}}\right) \\
& -\frac{1}{4 \sigma^{2}} \psi^{\alpha}\left(\bar{\sigma}^{m}\right)_{\alpha \dot{\alpha}} \bar{\psi}^{\dot{\alpha}}(* H)_{m}-\frac{1}{4 \sqrt{2} \sigma^{3}} \psi^{\alpha} \psi_{\alpha} \bar{\psi}_{\dot{\alpha}} \bar{\psi}^{\dot{\alpha}}-\frac{1}{4} F^{m n} F_{m n}-\frac{i}{2}\left(\bar{\lambda}^{\alpha}\left(\sigma^{m}\right)_{\alpha \dot{\alpha}} \partial_{m} \bar{\lambda}^{\dot{\alpha}}+\bar{\lambda}_{\dot{\alpha}}\left(\bar{\sigma}^{m}\right)^{\dot{\alpha} \alpha} \partial_{m} \lambda_{\alpha}\right)+\frac{1}{2} \boldsymbol{D}^{2}-\frac{1}{2} \xi \boldsymbol{D} \\
& +\frac{e}{2 \cdot 2 ! \cdot 2 !} \epsilon^{m n p q} B_{m n} F_{p q}+\frac{i e}{\sqrt{2}}\left(\lambda^{\alpha} \psi_{\alpha}-\bar{\lambda}_{\dot{\alpha}} \bar{\psi}^{\dot{\alpha}}\right)+2 \sqrt{2} e \sigma \boldsymbol{D}-\frac{1}{\sqrt{2}}\left(\psi^{\alpha} j_{\alpha}+\bar{\psi}_{\dot{\alpha}} \bar{j}^{\dot{\alpha}}\right)+\frac{1}{2 \cdot 2 !} \tilde{J}^{m n} B_{m n}+\sqrt{2} \sigma J
\end{aligned}
$$

Here, the components of the chiral superfield $\Sigma_{\alpha}$ and the linear sueprfield $L$ are denoted as

$$
\begin{aligned}
B_{m n} & =-i\left(\left(\sigma_{m n}\right)_{\alpha}{ }^{\beta} D^{\alpha} \Sigma_{\beta}-\left(\bar{\sigma}_{m n}\right)^{\dot{\alpha}} \bar{D}_{\dot{\alpha}} \bar{\Sigma}^{\dot{\beta}}\right) \\
H_{m n p} & =\partial_{m} B_{n p}+\partial_{n} B_{p m}+\partial_{p} B_{m n}=\frac{1}{4} \epsilon_{m n p q}\left(\bar{\sigma}^{q}\right)^{\dot{\alpha} \beta}\left[D_{\beta}, \bar{D}_{\dot{\alpha}}\right] L \\
\sigma & :=\frac{1}{\sqrt{2}} L\left|, \quad \psi_{\alpha}:=\frac{1}{\sqrt{2}} D_{\alpha} L\right|=+\frac{i}{4 \sqrt{2}} D^{2} \Sigma_{\alpha} \mid \\
\bar{\psi}_{\dot{\alpha}} & :=\frac{1}{\sqrt{2}} \bar{D}_{\dot{\alpha}} L\left|=-\frac{i}{4 \sqrt{2}} \bar{D}^{2} \bar{\Sigma}_{\dot{\alpha}}\right| .
\end{aligned}
$$

The vector component can also be written as

$$
(* H)^{m}=\frac{1}{3 !} \epsilon^{m n p q} H_{n p q}=\frac{1}{4}\left(\bar{\sigma}^{m}\right)^{\dot{\alpha} \beta}\left[D_{\beta}, \bar{D}_{\dot{\alpha}}\right] L,
$$

or

$$
\left[D_{\alpha}, \bar{D}_{\dot{\alpha}}\right] L \mid=-2(* H)_{\alpha \dot{\alpha}} .
$$

Note that we have used the WZ gauge for the 2-form prepotential $\Sigma_{\alpha}$ :

$$
\Sigma_{\alpha}\left|=\bar{\Sigma}_{\dot{\alpha}}\right|=\left(D^{\alpha} \Sigma_{\alpha}+\bar{D}_{\dot{\alpha}} \bar{\Sigma}^{\dot{\alpha}}\right) \mid=0 .
$$

Note that the superparters of the phase of the complex scalar field are also dualized to the 2 -form prepotential in the Lagrangian in Eq. (3.24) due to SUSY in contrast to the bosonic case. In the Lagrangian in Eq. (3.24), the fields $j_{\alpha}$, $\bar{j}_{\dot{\alpha}}, J_{m n}$, and $J$ are the components of $J_{\alpha}$ :

$$
\begin{aligned}
j_{\alpha} & =J_{\alpha}\left|, \quad \bar{j}_{\dot{\alpha}}=\bar{J}_{\dot{\alpha}}\right|, \\
J_{m n} & =\frac{1}{2 i}\left(\left(\sigma_{m n}\right)_{\alpha}^{\beta} D^{\alpha} J_{\beta}-\left(\bar{\sigma}_{m n}\right)^{\dot{\alpha}} \bar{D}_{\dot{\alpha}} \bar{J}^{\dot{\beta}}\right) \mid, \\
\tilde{J}_{m n} & =\frac{1}{2 !} \epsilon_{m n p q} J^{p q}, \\
J & =-\frac{1}{2} D^{\alpha} J_{\alpha}\left|=-\frac{1}{2} \bar{D}_{\dot{\alpha}} \bar{J}^{\dot{\alpha}}\right| .
\end{aligned}
$$

It seems that $J_{\alpha}=0$ since $\log \left|\Phi_{0}\right|^{2}=\log \Phi_{0}+\log \bar{\Phi}_{0}$. However, since $\Phi_{0}$ can have zero points, $\bar{D}^{2} D_{\alpha} \log \left|\Phi_{0}\right|^{2}$ should contain a term like a delta function as mentioned above. The delta function arises as a SUSY extension of Eq. (2.12),

$\frac{\partial}{\partial \Phi_{0}} \frac{\partial}{\partial \bar{\Phi}_{0}} \log \left|\Phi_{0}\right|^{2}=\frac{\partial}{\partial \Phi_{0}} \frac{1}{\bar{\Phi}_{0}}=\frac{\partial}{\partial \bar{\Phi}_{0}} \frac{1}{\Phi_{0}}=2 \pi \delta^{2}(\Phi, \bar{\Phi})$,

where $\delta^{2}(\Phi, \bar{\Phi})$ is defined by

$$
\delta^{2}(\Phi, \bar{\Phi})=\frac{1}{2} \delta(\operatorname{Re} \Phi) \delta(\operatorname{Im} \Phi)
$$


Note that this property of $\log \left|\Phi_{0}\right|^{2}$ can also be understood as a SUSY extension of the two-dimensional Green's function. We explicitly write down the components of $J_{\alpha}$ as follows:

$$
\begin{aligned}
j_{\alpha}= & \left.J_{\alpha}\left|=-\frac{1}{4} \bar{D}^{2} D_{\alpha} \log \right| \Phi_{0}\right|^{2} \mid \\
= & 2 \sqrt{2} \pi \delta^{2}\left(\phi_{0}, \bar{\phi}_{0}\right) \bar{F}_{0} \chi_{0 \alpha} \\
& -2 \sqrt{2} i \pi \delta^{2}\left(\phi_{0}, \bar{\phi}_{0}\right)\left(\bar{\sigma}^{m}\right)_{\alpha \dot{\alpha}} \partial_{m} \phi_{0} \bar{\chi}^{0 \dot{\alpha}} \\
& -\sqrt{2} \pi\left(\frac{\partial}{\partial \bar{\phi}_{0}} \delta^{2}\left(\phi_{0}, \bar{\phi}_{0}\right)\right) \bar{\chi}_{0 \dot{\alpha}} \bar{\chi}_{0}^{\dot{\alpha}} \chi_{0 \alpha} .
\end{aligned}
$$

Here, $\phi_{0}, \chi_{0}$, and $F_{0}$ are defined by

$\phi_{0}=\Phi_{0}\left|, \quad \chi_{0 \alpha}=\frac{1}{\sqrt{2}} D_{\alpha} \Phi_{0}\right|, \quad F_{0}=-\frac{1}{4} D^{2} \Phi_{0} \mid$,

and we have used

$$
D_{\alpha} \log \left|\Phi_{0}\right|^{2}=\left(D_{\alpha} \Phi_{0}\right) \frac{\partial}{\partial \Phi_{0}} \log \left|\Phi_{0}\right|^{2}
$$

and its Hermitian conjugate. The component $J$ can also be rewritten as

$$
\begin{aligned}
J= & -\frac{1}{2} D^{\alpha} J_{\alpha}\left|=-\frac{1}{2} \bar{D}_{\dot{\alpha}} \bar{J}^{\dot{\alpha}}\right|=\frac{1}{8} D^{\alpha} \bar{D}^{2} D_{\alpha} \log \left|\Phi_{0}\right|^{2} \mid \\
= & 4 \pi \delta^{2}\left(\phi_{0}, \bar{\phi}_{0}\right)\left(-\partial_{m} \phi_{0} \partial^{m} \bar{\phi}_{0}-\frac{i}{2} \chi_{0}^{\alpha}\left(\sigma^{n}\right)_{\alpha \dot{\alpha}} \partial_{n} \bar{\chi}_{0}^{\dot{\alpha}}-\frac{i}{2} \bar{\chi}_{0 \dot{\alpha}}\left(\sigma^{n}\right)^{\dot{\alpha} \alpha} \partial_{n} \chi_{0 \alpha}+F_{0} \bar{F}_{0}\right) \\
& +2 i \pi\left(\frac{\partial}{\partial \bar{\phi}_{0}} \delta^{2}\left(\phi_{0}, \bar{\phi}_{0}\right) \partial_{m} \bar{\phi}_{0}-\frac{\partial}{\partial \phi_{0}} \delta^{2}\left(\phi_{0}, \bar{\phi}_{0}\right) \partial_{m} \phi_{0}\right) \bar{\chi}_{0 \dot{\alpha}}\left(\bar{\sigma}^{m}\right)^{\alpha \dot{\alpha}} \chi_{0 \alpha} \\
& -2 \pi\left(\frac{\partial}{\partial \bar{\phi}_{0}} \delta^{2}\left(\phi_{0}, \bar{\phi}_{0}\right)\right) \bar{\chi}_{0 \dot{\alpha}} \bar{\chi}_{0}^{\dot{\alpha}} F_{0}-2 \pi\left(\frac{\partial}{\partial \phi_{0}} \delta^{2}\left(\phi_{0}, \bar{\phi}_{0}\right)\right) \chi_{0}^{\alpha} \chi_{0 \alpha} \bar{F}_{0}+\pi\left(\frac{\partial}{\partial \phi_{0}} \frac{\partial}{\partial \bar{\phi}_{0}} \delta^{2}\left(\phi_{0}, \bar{\phi}_{0}\right)\right) \chi_{0}^{\alpha} \chi_{0 \alpha \alpha} \bar{\chi}_{0 \dot{\alpha}} \bar{\chi}_{0}^{\dot{\alpha}} .
\end{aligned}
$$

This component may correspond to (twice) the Lagrangian of the nonlinear sigma model in which the Kähler potential is given by $K=\log \left|\Phi_{0}\right|^{2}$. Finally, the component $\tilde{J}_{m n}=\frac{1}{2 !} \epsilon_{m n p q}{ }^{p q}$ can be calculated as

$$
\begin{aligned}
\tilde{J}_{m n}= & -4 i \pi \delta^{2}\left(\phi_{0}, \bar{\phi}_{0}\right) \epsilon_{m n p q} \partial^{p} \phi_{0} \partial^{q} \bar{\phi}_{0}-2 \pi \delta^{2}\left(\phi_{0}, \bar{\phi}_{0}\right) \epsilon_{m n p q}\left(\chi_{0}^{\alpha}\left(\sigma^{q}\right)_{\alpha \dot{\alpha}}\left(\partial^{p} \bar{\chi}_{0}^{\dot{\alpha}}\right)-\bar{\chi}_{0 \dot{\alpha}}\left(\bar{\sigma}^{q}\right)^{\dot{\alpha} \beta} \partial^{p} \chi_{0 \beta}\right) \\
& +2 \pi \epsilon_{m n p q}\left(\frac{\partial}{\partial \bar{\phi}_{0}} \delta^{2}\left(\phi_{0}, \bar{\phi}_{0}\right) \partial^{p} \bar{\phi}_{0}+\frac{\partial}{\partial \phi_{0}} \delta^{2}\left(\phi_{0}, \bar{\phi}_{0}\right) \partial^{p} \phi_{0}\right) \bar{\chi}_{0 \dot{\alpha}}\left(\bar{\sigma}^{q}\right)^{\dot{\alpha} \alpha} \chi_{0 \alpha} .
\end{aligned}
$$

Since the right-hand side of the first line in Eq. (3.36) corresponds to Eq. (2.11) in the bosonic case, $\tilde{J}_{m n}$ can be understood as a SUSY extension of the string current. The conservation law of $\tilde{J}_{m n}$ can be derived by the relation $D^{\alpha} J_{\alpha}=\bar{D}_{\dot{\alpha}} \bar{J}^{\dot{\alpha}}$ in Eq. (3.23), which implies

$$
\partial_{m} \tilde{J}^{m n}=0
$$

Note that Eq. (3.38) is equivalent to the property that $J_{m n}$ is closed:

$$
\epsilon^{m n p q} \partial_{n} J_{p q}=0
$$

Before closing this section, a comment is in order on the string current superfield. Since Eqs. (3.23) and (3.38) hold, the string current superfield can be a SUSY extension of the closed 2-form, which cannot be expressed by the exterior derivative of a regular 1-form. If we regard the string current superfield as a "gaugino superfield" of a singular 2-form field strength, the "prepotential" for the gaugino superfield may correspond to $\log \left|\Phi_{0}\right|^{2}$. In this case, the vector component of $\log \left|\Phi_{0}\right|^{2}$ is singular at the zero points of $\phi_{0}$ :

$$
\begin{aligned}
& \left(\left[D_{\alpha}, \bar{D}_{\dot{\alpha}}\right] \log \left|\Phi_{0}\right|^{2}\right) \mid \\
& =-2 i\left(\sigma^{m}\right)_{\alpha \dot{\alpha}}\left(\frac{1}{\bar{\phi}_{0}} \partial_{m} \bar{\phi}_{0}-\frac{1}{\phi_{0}} \partial_{m} \phi_{0}\right)-8 \pi \delta^{2}\left(\phi_{0}, \bar{\phi}_{0}\right) \chi_{0 \alpha} \bar{\chi}_{0 \dot{\alpha}} .
\end{aligned}
$$

\section{String charge in original and dual formulations}

While we have obtained the string current superfield $J_{\alpha}$ in the dual formulation, it is still unclear whether $J_{\alpha}$ recovers the vortex string in the original formulation. Here, we show that the string charge, which is obtained by $J_{\alpha}$ in the dual theory, is equal to the string tensorial charge in the original theory. We focus on the configuration such that all the fermionic excitations are negligible.

In the original formulation, the string tensorial charge $Z^{m}$ is saturated by the configuration of the 1-form gauge field [52], 


$$
Z^{m}=\xi \int d^{3} \boldsymbol{x} \epsilon^{0 m n p} \partial_{n} A_{p}
$$

where $\int d^{3} \boldsymbol{x}$ denotes a three-dimensional spatial integration. Note that the quantity $Z^{m}$ is defined in the original formulation, while $Z^{m}$ itself can make sense in the dual formulation because the 1-form gauge field has not been dualized.

On the one hand, in the original formulation, the tensorial charge can be evaluated as follows. Since the integrand is a total derivative, we can evaluate the tensorial charge as a configuration of the gauge field $A_{m}$ at an infinitely large distance from vortices. We require a boundary condition for the scalar field $\phi$ such that the covariant derivative on the scalar field $\partial_{m} \phi-\frac{i e}{2} A_{m} \phi$ should vanish at an infinitely large distance from vortices in order to realize a configuration with a finite energy per unit length of the vortices. By this condition, the 1-form gauge field $A_{m}$ is related to the derivative $\partial_{m} \phi$ as

$$
A_{m} \rightarrow A_{m}^{\infty}=\frac{1}{2 i} \cdot \frac{2}{e} \partial_{m} \log (\phi / \bar{\phi}) .
$$

Therefore, the string tensorial charge can be expressed as

$$
\begin{aligned}
Z^{m} & =\xi \int d^{3} \boldsymbol{x} \epsilon^{0 m n p} \partial_{n} A_{p}^{\infty} \\
& =\frac{\xi}{2 i} \cdot \frac{2}{e} \int d^{3} \boldsymbol{x} \epsilon^{0 m n p} \partial_{n} \partial_{p} \log (\phi / \bar{\phi}) .
\end{aligned}
$$

By using Eq. (2.11), the string tensorial charge can be now expressed as a configuration of the vortices,

$$
Z^{m}=4 \pi i \frac{\xi}{e} \int d^{3} \boldsymbol{x} \epsilon^{0 m n p} \delta^{2}\left(\phi_{0}, \bar{\phi}_{0}\right) \partial_{n} \phi_{0} \partial_{p} \bar{\phi}_{0},
$$

where we have used $\epsilon^{m n p q} \partial_{n} \partial_{p} \log (\phi / \bar{\phi})=\epsilon^{m n p q} \partial_{n} \partial_{p}$ $\log \left(\phi_{0} / \bar{\phi}_{0}\right)$, since only the zero points of the scalar field $\phi_{0}$ contribute to the singularities.

On the other hand, in the dual formulation, we try to construct the quantity $Z^{\prime m}$, which corresponds to the tensorial charge in the original formulation:

$$
Z^{\prime m}=\xi \int d^{3} x \epsilon^{0 m n p} \partial_{n} A_{p} .
$$

In the dual formulation, the configuration of the 1-form gauge field is given by the 2-form gauge field $B_{m n}$ and the string current $\widetilde{J}^{m n}$ rather than the configuration of the scalar field. The configuration can be seen by the EOM for the 2-form gauge field:

$$
\frac{e}{2} \tilde{F}^{m n}=-\frac{1}{2} \tilde{J}^{m n}+\partial_{q}\left(\frac{1}{\sqrt{2} \sigma} H^{q m n}\right) .
$$

Substituting the equation into Eq. (3.40), we obtain string charge in the dual formulation:

$Z^{\prime m}=\frac{2 \xi}{e} \int d^{3} \boldsymbol{x}\left(-\frac{1}{2} \tilde{J}^{0 m}+\partial_{q}\left(\frac{1}{\sqrt{2} \sigma} H^{q 0 m}\right)\right)$.

The second term in the right-hand side is a surface integral. This surface term vanishes by the following boundary conditions, which are required by the finiteness of the energy per unit length of the vortices. At a large-distance limit, the scalar field $\sigma$ approaches the minimum of the potential of $\sigma, \sigma \rightarrow \frac{\sqrt{2} \xi}{4 e}$, and the field strength $H_{m n p}$ goes to zero, $H_{m n p} \rightarrow 0$. Therefore, the string charge is saturated by the string current $J_{m n}$. By using Eq. (3.36), we find that the string charge in the dual formulation is equal to the tensorial charge in the original formulation in Eq. (3.43):

$$
\begin{aligned}
Z^{\prime m} & =-\frac{\xi}{e} \int d^{3} \boldsymbol{x} \tilde{J}^{0 m} \\
& =4 \pi i \frac{\xi}{e} \int d^{3} \boldsymbol{x} \epsilon^{0 m n p} \delta^{2}\left(\phi_{0}, \bar{\phi}_{0}\right) \partial_{n} \phi_{0} \partial_{p} \bar{\phi}_{0}=Z^{m} .
\end{aligned}
$$

Therefore, the string current $J_{m n}$ consistently reproduces the string charge, which corresponds to the tensorial charge in the original formulation.

\section{Dual SUSY massive 2-form theory with vortex strings}

Here, we further dualize the Lagrangian in Eq. (3.21). The Lagrangian in Eq. (3.21) is described by the 1-form prepotential $V$ and the 2 -form prepotential $\Sigma_{\alpha}$ with the topological coupling $\epsilon^{m n p q} B_{m n} F_{p q}$. In this picture, the string current superfield is coupled with the 2 -from prepotential in a gauge-invariant way. We can further dualize the Lagrangian as we will see below. In this picture, the 2-form gauge field is manifestly massive since the 2-form gauge field eats the 1-form gauge field by the Stückelberg mechanism. The dual transformation can be done by adding a Lagrange multiplier $\Upsilon_{\alpha}$ in the Lagrangian:

$$
\begin{aligned}
\mathcal{L}_{B^{\prime}, \mathrm{SUSY}, 1 \mathrm{st}}^{\prime}= & -\frac{1}{2} \int d^{4} \theta L \log \left(\frac{L}{M^{2}}\right)+\frac{1}{4} \int d^{2} \theta W^{\prime \alpha} W_{\alpha}^{\prime} \\
& +\frac{1}{2} \int d^{4} \theta(e L-\xi) V-\frac{1}{2 i} \int d^{2} \theta \Sigma^{\alpha} J_{\alpha} \\
& -\frac{1}{2 i} \int d^{2} \theta \Upsilon^{\alpha}\left(W_{\alpha}^{\prime}+\frac{1}{4} \bar{D}^{2} D_{\alpha} V\right)+\text { H.c. }
\end{aligned}
$$

Here, $\Upsilon_{\alpha}$ is a chiral superfield as a Lagrange multiplier, and $W_{\alpha}^{\prime}$ is a chiral superfield which is independent of the real superfield $V$. Note that $\Upsilon_{\alpha}$ do not have a gauge symmetry, in contrast to $\Sigma_{\alpha}$. The EOM for $\Upsilon_{\alpha}$ gives us the original Lagrangian as before, while the EOM for $W_{\alpha}^{\prime}$ gives us 


$$
W_{\alpha}^{\prime}=-i \Upsilon_{\alpha}
$$

This equation implies that $W_{\alpha}^{\prime}$ is now described by the chiral superfield $\Upsilon_{\alpha}^{\prime}$. Further, the EOM for the 1-form prepotential $V$ leads to

$$
L=\frac{1}{e}(\Psi+\xi),
$$

where $\Psi$ is given by

$$
\Psi:=\frac{1}{2 i}\left(D^{\alpha} \Upsilon_{\alpha}-\bar{D}_{\dot{\alpha}} \bar{\Upsilon}^{\dot{\alpha}}\right) .
$$

The relation in Eq. (3.50) means that the 2-form prepotential $\Sigma_{\alpha}$ can be described by the chiral superfield $\Upsilon_{\alpha}$. Substituting Eqs. (3.49) and (3.50) into the Lagrangian in Eq. (3.48), we obtain the following dual Lagrangian:

$$
\begin{aligned}
\mathcal{L}_{B^{\prime}, \mathrm{SUSY}}^{\prime}= & -\frac{1}{2 e} \int d^{4} \theta(\Psi+\xi) \log \left(\frac{\Psi+\xi}{e M^{2}}\right)+\frac{1}{4} \int d^{2} \theta \Upsilon^{\alpha} \Upsilon_{\alpha} \\
& +\frac{\xi}{2 e} \int d^{4} \theta \log \left|\Phi_{0}\right|^{2}-\frac{1}{2 i e} \int d^{2} \theta \Upsilon^{\alpha} J_{\alpha}+\text { H.c. }
\end{aligned}
$$

The Lagrangian is now given by the chiral superfields $\Upsilon_{\alpha}$ and $\Phi_{0}$. The chiral superfield $\Upsilon_{\alpha}$ describes a massive 2 -form and its superpartners. The first term is the kinetic term for the massive 2-form, and the second term is the mass term. The third and the fourth terms are the coupling between the massive 2-form superfield and the string current superfield. These terms can be explicitly seen by the component expression of the Lagrangian in Eq. (3.52).
To show the component Lagrangian, we define the component fields of the chiral spinor superfield $\Upsilon_{\alpha}$ as follows. The $\theta=\bar{\theta}=0$ components are defined as

$$
\lambda_{\alpha}^{\prime}=+i \Upsilon_{\alpha}\left|, \quad \bar{\lambda}_{\dot{\alpha}}^{\prime}=-i \bar{\Upsilon}_{\dot{\alpha}}\right| .
$$

The components given by first-order spinor derivatives are

$$
\begin{aligned}
D^{\prime} & =-\frac{1}{4}\left(D^{\alpha} \Upsilon_{\alpha}+\bar{D}_{\dot{\alpha}} \bar{\Upsilon}^{\dot{\alpha}}\right) \mid, \\
\sigma^{\prime} & =\frac{1}{\sqrt{2}} \Psi\left|=\frac{1}{2 \sqrt{2} i}\left(D^{\alpha} \Upsilon_{\alpha}-\bar{D}_{\dot{\alpha}} \bar{\Upsilon}^{\dot{\alpha}}\right)\right|, \\
B_{m n}^{\prime} & =-i\left(\left(\sigma_{m n}\right)_{\alpha}{ }^{\beta} D^{\alpha} \Upsilon_{\beta}-\left(\bar{\sigma}_{m n}\right)^{\dot{\alpha}}{ }_{\dot{\beta}} \bar{D}_{\dot{\alpha}} \bar{\Upsilon}^{\dot{\beta}}\right) \mid .
\end{aligned}
$$

Here, $B_{m n}^{\prime}$ is a (nongauge) 2 -form field. The components defined by second-order spinor derivatives are

$$
\begin{aligned}
\psi_{\alpha}^{\prime} & :=\frac{1}{\sqrt{2}} D_{\alpha} \Psi\left|=+\frac{i}{4 \sqrt{2}} D^{2} \Upsilon_{\alpha}\right|+\partial_{\alpha \dot{\beta}} \bar{\Upsilon}^{\dot{\beta}} \mid, \\
\bar{\psi}^{\prime \dot{\alpha}} & :=\frac{1}{\sqrt{2}} \bar{D}^{\dot{\alpha}} \Psi\left|=-\frac{i}{4 \sqrt{2}} \bar{D}^{2} \bar{\Upsilon}^{\dot{\alpha}}\right|-\partial^{\dot{\alpha} \beta} \Upsilon_{\beta} \mid .
\end{aligned}
$$

Since $\Upsilon_{\alpha}$ is a chiral superfield, the components of higher than the second order are given by spacetime derivatives of the lower components. For example, the exterior derivative on the 2-form field is expressed in terms of the superfield as follows:

$$
\begin{aligned}
H_{m n p}^{\prime} & :=\partial_{m} B_{n p}^{\prime}+\partial_{n} B_{p m}^{\prime}+\partial_{p} B_{m n}^{\prime} \\
& =\frac{1}{4} \epsilon_{m n p q}\left(\bar{\sigma}^{q}\right)^{\dot{\alpha} \beta}\left[D_{\beta}, \bar{D}_{\dot{\alpha}}\right] \Psi \mid .
\end{aligned}
$$

By using these component fields, we obtain the component Lagrangian:

$$
\begin{aligned}
\mathcal{L}_{B^{\prime}, \text { SUSY }}^{\prime}= & -\frac{1}{2 e\left(\sqrt{2} \sigma^{\prime}+\xi\right)}\left(\left(\partial^{m} \sigma^{\prime}\right)\left(\partial_{m} \sigma^{\prime}\right)-\left(* H^{\prime}\right)^{m}\left(* H^{\prime}\right)_{m}\right)-\frac{i}{2 e\left(\sqrt{2} \sigma^{\prime}+\xi\right)}\left(\bar{\psi}_{\dot{\alpha}}^{\prime}\left(\bar{\sigma}^{m}\right)^{\dot{\alpha} \alpha} \partial_{m} \psi_{\alpha}^{\prime}+\psi^{\prime \alpha}\left(\sigma^{m}\right)_{\alpha \dot{\alpha}} \partial_{m} \bar{\psi}^{\prime} \dot{\alpha}\right) \\
& -\frac{1}{2 e\left(\sqrt{2} \sigma^{\prime}+\xi\right)^{2}} \psi^{\prime \alpha}\left(\bar{\sigma}^{m}\right)_{\alpha \dot{\alpha}} \bar{\psi}^{\prime \dot{\alpha}}\left(* H^{\prime}\right)_{m}-\frac{1}{2 e\left(\sqrt{2} \sigma^{\prime}+\xi\right)^{3}} \psi^{\prime \alpha} \psi_{\alpha}^{\prime} \bar{\psi}_{\dot{\alpha}}^{\prime} \bar{\psi}^{\prime \dot{\alpha}}-\frac{1}{16} B^{\prime m n} B_{m n}^{\prime}+\frac{1}{2} \boldsymbol{D}^{\prime 2}-\frac{1}{4} \sigma^{\prime 2} \\
& -\frac{i}{2}\left(\lambda^{\prime \beta}\left(\sigma^{m}\right)_{\beta \dot{\beta}} \partial_{m} \bar{\lambda}^{\prime \dot{\beta}}+\bar{\lambda}_{\dot{\beta}}^{\prime}\left(\bar{\sigma}^{m}\right)^{\dot{\beta} \beta} \partial_{m} \lambda_{\beta}^{\prime}\right)-\frac{1}{\sqrt{2}} i\left(\lambda^{\prime \alpha} \psi_{\alpha}^{\prime}-\bar{\lambda}_{\dot{\alpha}}^{\prime} \bar{\psi}^{\prime \dot{\alpha}}\right)+\frac{1}{2 \cdot 2 ! e} \tilde{J}^{m n} B_{m n}^{\prime}+\frac{1}{2 e}(\sqrt{2} \sigma+\xi) J \\
& -\frac{1}{\sqrt{2} e}\left(j^{\alpha} \psi_{\alpha}^{\prime}+\bar{j}_{\dot{\alpha}} \bar{\psi}^{\prime \dot{\alpha}}\right)+\frac{i}{2 e}\left(j^{\alpha}\left(\sigma^{m}\right)_{\alpha \dot{\beta}} \partial_{m} \bar{\lambda}^{\prime} \dot{\beta}+\bar{j}_{\dot{\alpha}}\left(\bar{\sigma}^{m}\right)^{\dot{\alpha} \beta} \partial_{m} \lambda_{\beta}^{\prime}\right) .
\end{aligned}
$$

The term $B^{\prime m n} B_{m n}^{\prime}$ is the mass term for the 2 -form field. The coupling between the 2-form field and the string current is represented by $\tilde{J}^{m n} B_{m n}^{\prime}$. In this Lagrangian, we find that there are couplings between the fermionic component of the string current superfield $j_{\alpha}$ and $\lambda_{\alpha}^{\prime}$ compared with the Lagrangian in Eq. (3.24). Note that the superpartners of the 1 -form gauge field are also dualized to the chiral spinor superfield in the Lagrangian in Eq. (3.57) due to SUSY, similarly to the Lagrangian in Eq. (3.24).

\section{SUMMARY}

In this paper, we have derived the dual formulations of the SUSY Abelian Higgs model with the FI term in four 
dimensions. In particular, we have focused on the dual transformations of ANO vortex strings in $\mathcal{N}=1$ superspace. These formulations of the ANO vortex strings can be obtained by splitting the chiral superfield charged under the $U(1)$ gauge symmetry into the regular part and singular part. For the regular part, which does not have zero points, we have dualized this part into a 2 -form prepotential in a previously known way. In both of the dual formulations, the superpartners of the phase of the scalar field and 1-form are dualized into the 2-form prepotential and chiral spinor superfield due to SUSY, in contrast to the bosonic case, respectively.

In the dual transformation to the system with the 2-form prepotential, we have shown that the singular part of the chiral superfield gives us the string current superfield which has singularities of the two-dimensional delta function. The string current superfield is coupled with the 2-form prepotential or the chiral spinor superfield and satisfies the current conservation law by the SUSY algebra. This current conservation law is consistent with the gauge symmetry of the 2-form prepotential. Furthermore, we have identified the components of the string current superfield. There are vortex strings as well as their superpartners. We have confirmed that the vortex strings in the string current superfield are the same as the ones in the bosonic (non-SUSY) Abelian Higgs model.

We have further dualized the Abelian Higgs model into a theory described by a massive 2-form field. The dual transformation has also been obtained by the previously known way. We have also shown that the string current superfield is coupled with the chiral spinor superfield into which the massive 2-form field is embedded.

There are several avenues for future work? One is the BPS conditions for the ANO vortex strings in the dual formulations. We have not considered the BPS conditions for the ANO vortex strings, although the conditions are important in SUSY theories. Thus, we should discuss the dualities of the BPS conditions on the ANO vortex strings. Another is the dual formulations including superpotentials, which uplift flat directions of the D-term potential. In particular, we may discuss the dual formulations of the so-called $\mathrm{M}$ model $[53,54]$, in which the D-term potential is uplifted by a superpotential with an additional neutral chiral superfield.

The physical meaning of the bosonic and the fermionic superpartners of the string current should also be investigated. These superpartners are defined by spinor derivatives of the singular part of the chiral superfield. They are coupled with the superpartners of the 2-form prepotential or the chiral spinor superfield of the massive 2-form. It may be an open question whether such couplings are particular ones for SUSY theories or can be generalized to nonSUSY cases.

Mathematical structures of the string current superfield would be interesting. In $4 \mathrm{D} \mathcal{N}=1$ SUSY theories, the superspace expressions of closed or exact $p$-forms have been already known [44]. On the other hand, the string current superfield formulated in this paper can be an example of a superspace extension of the closed 2-form $J_{m n}$, which cannot be expressed by an exterior derivative of a globally well-defined 1-form. The generalization of such properties of the string current superfield to other $p$-forms may be useful to discuss other topological solitons.

The SUSY Abelian Higgs model is the simplest Lagrangian consisting of a single vector superfield and a single chiral superfield. When such a theory is realized as a low-energy effective action, it usually contains higherderivative corrections. Ghost-free higher-derivative terms for a vector superfield and chiral superfield are available in Ref. [55] and Refs. [56-66], respectively. An extension of our duality with vortex strings in more general cases with higher derivative terms is one of future directions.

The dual transformations discussed in this paper can be extended to cosmic strings in SUGRA [40-42]. It will be convenient to use conformal SUGRA [67-73] when we discuss the dual transformations of ANO vortex strings, since the canonically normalized Einstein-Hilbert term can be obtained by the superconformal gauge fixing without tedious super-Weyl rescalings [69]. In particular, the conformal superspace formalism [71] and $p$-form gauge theories in the conformal superspace $[74,75]$ would be useful, since we can discuss dual transformations in a manifestly SUSY way.

One of the important extensions would be a non-Abelian extension. A $U(N)$ gauge theory coupled with $N \times N$ Higgs fields in the fundamental representation with common $U(1)$ charges is known to admit a non-Abelian vortex accompanied with non-Abelian $\mathbb{C} P^{N-1}$ moduli [76-82]; see Refs. [32,54,83,84] as a review. A nonAbelian duality of a non-Abelian vortex in a non-SUSY case was done in the context of dense QCD [27,29], by using a non-Abelian 2 -form field $[23,24] .{ }^{1}$ There, a coupling between the $\mathbb{C} P^{N-1}$ fields localized on a vortex world sheet and a non-Abelian 2-form field in the bulk was obtained. A non-Abelian duality of non-Abelian vortex strings in a SUSY case would be possible by a non-Abelian extension of a chiral spinor superfield including a non-Abelian 2-form field as a component [86,87]. Another possibility of extensions is the case of an $S U(2) \times U(1)$ gauge theory coupled with the triplet Higgs fields with an equal charge, admitting a BPS Alice string [88,89]. This will be also possible by using a non-Abelian chiral spinor superfield.

It would be interesting to consider the dual transformations of $\mathcal{N}=2$ extended SUSY theories allowing ANO vortex strings as well as non-Abelian vortex strings [76-82]. To this end, the framework discussed in Ref. [49] might be useful. $\mathcal{N}=2$ extended SUSY theories also admit several composite solitons containing vortices such as vortex strings ending on a domain wall [90-92], a

\footnotetext{
${ }^{1}$ Instead of the full non-Abelian duality, a partial duality can be done by focusing on Abelian diagonal components [85].
} 
monopole confined by vortices [78,93-95], Yang-Mills instantons trapped inside a vortex [78,93,94], and intersecting vortex strings $[96,97]$. The dual transformations in the presence of these composite solitons would be one of interesting future directions. Along this line, a dual transformation of a vortex-monopole complex was already discussed in Ref. [98].

\section{ACKNOWLEDGMENTS}

R. Y. thanks Hiroshi Isono for helpful discussions. This work is supported by the Ministry of Education, Culture,
Sports, Science (MEXT-)Supported Program for the Strategic Research Foundation at Private Universities "Topological Science" (Grant No. S1511006). The work of M. N. is also supported in part by the Japan Society for the Promotion of Science (JSPS) Grant-in-Aid for Scientific Research (KAKENHI Grants No. 16H03984 and No. 18H01217). The work of M. N. and R. Y. is also supported in part by a Grant-in-Aid for Scientific Research on Innovative Areas "Topological Materials Science" (KAKENHI Grant No. 15H05855) and "Discrete Geometric Analysis for Materials Design" (KAKENHI Grant No. 17H06462) from MEXT of Japan, respectively.
[1] A. A. Abrikosov, On the Magnetic properties of superconductors of the second group, Zh. Eksp. Teor. Fiz. 32, 1442 (1957) [Sov. Phys. JETP 5, 1174 (1957)].

[2] H. B. Nielsen and P. Olesen, Vortex line models for dual strings, Nucl. Phys. B61, 45 (1973).

[3] A. Vilenkin, Gravitational field of vacuum domain walls and strings, Phys. Rev. D 23, 852 (1981).

[4] A. Vilenkin, Cosmological Density Fluctuations Produced by Vacuum Strings, Phys. Rev. Lett. 46, 1169 (1981); Erratum, Phys. Rev. Lett. 46, 1496(E) (1981).

[5] A. Vilenkin, Cosmic strings, Phys. Rev. D 24, 2082 (1981).

[6] A. Vilenkin, Cosmic strings and domain walls, Phys. Rep. 121, 263 (1985).

[7] A. Vilenkin and E. P.S. Shellard, Cosmic Strings and Other Topological Defects (Cambridge University Press, Cambridge, England, 2000).

[8] K. Hayashi, The $A_{1}$ and mesons from the violation of local Lorentz invariance, Phys. Lett. 44B, 497 (1973).

[9] E. Cremmer and J. Scherk, Spontaneous dynamical breaking of gauge symmetry in dual models, Nucl. Phys. B72, 117 (1974).

[10] M. Kalb and P. Ramond, Classical direct interstring action, Phys. Rev. D 9, 2273 (1974).

[11] Y. Nambu, Strings, monopoles and gauge fields, Phys. Rev. D 10, 4262 (1974).

[12] G. T. Horowitz, Exactly soluble diffeomorphism invariant theories, Commun. Math. Phys. 125, 417 (1989).

[13] T. J. Allen, M. J. Bowick, and A. Lahiri, Topological mass generation in $(3+1)$-dimensions, Mod. Phys. Lett. A 06, 559 (1991).

[14] M. Henneaux and C. Teitelboim, P form electrodynamics, Found. Phys. 16, 593 (1986).

[15] K.-M. Lee, The dual formulation of cosmic strings and vortices, Phys. Rev. D 48, 2493 (1993).

[16] Y. Takahashi and R. Palmer, Gauge-independent formulation of a massive field with spin one, Phys. Rev. D 1, 2974 (1970).

[17] A. Sugamoto, Dual transformation in Abelian gauge theories, Phys. Rev. D 19, 1820 (1979).
[18] H. Kawai, A dual transformation of the Nielsen-Olesen model, Prog. Theor. Phys. 65, 351 (1981).

[19] R. O. Ramos, J. F. Medeiros Neto, D. G. Barci, and C. A. Linhares, Abelian Higgs model effective potential in the presence of vortices, Phys. Rev. D 72, 103524 (2005).

[20] H. Kleinert, Multivalued fields, in Condensed Matter, Electromagnetism, and Gravitation (World Scientific, Singapore, 2008), p. 524.

[21] R. L. Davis, Spinning vortices in type II superconductors, Mod. Phys. Lett. A 05, 955 (1990).

[22] R. L. Davis and E. P. S. Shellard, Global Strings and Superfluid Vortices, Phys. Rev. Lett. 63, 2021 (1989).

[23] K. Seo, M. Okawa, and A. Sugamoto, Dual transformation in Nonabelian gauge theories, Phys. Rev. D 19, 3744 (1979).

[24] D. Z. Freedman and P. K. Townsend, Antisymmetric tensor gauge theories and nonlinear sigma models, Nucl. Phys. B177, 282 (1981).

[25] A. P. Balachandran, S. Digal, and T. Matsuura, Semi-superfluid strings in high density QCD, Phys. Rev. D 73, 074009 (2006).

[26] M. Eto and M. Nitta, Color magnetic flux tubes in dense QCD, Phys. Rev. D 80, 125007 (2009).

[27] M. Eto, Y. Hirono, M. Nitta, and S. Yasui, Vortices and other topological solitons in dense quark matter, Prog. Theor. Exp. Phys. 2014, 12D01 (2014).

[28] E. Nakano, M. Nitta, and T. Matsuura, Non-Abelian strings in high density QCD: Zero modes and interactions, Phys. Rev. D 78, 045002 (2008).

[29] Y. Hirono, T. Kanazawa, and M. Nitta, Topological interactions of Non-Abelian vortices with Quasi-particles in high density QCD, Phys. Rev. D 83, 085018 (2011).

[30] E. B. Bogomolny, Stability of classical solutions, Yad. Fiz. 24, 861 (1976) [Sov. J. Nucl. Phys. 24, 449 (1976)].

[31] M. K. Prasad and C. M. Sommerfield, An Exact Classical Solution for the 't Hooft Monopole and the Julia-Zee Dyon, Phys. Rev. Lett. 35, 760 (1975).

[32] M. Shifman and A. Yung, Supersymmetric Solitons, Cambridge Monographs on Mathematical Physics (Cambridge University Press, Cambridge, England, 2009). 
[33] H. J. de Vega and F. A. Schaposnik, A classical vortex solution of the Abelian Higgs model, Phys. Rev. D 14, 1100 (1976).

[34] E. Witten and D. I. Olive, Supersymmetry algebras that include topological charges, Phys. Lett. 78B, 97 (1978).

[35] P. Fayet, Higgs model and supersymmetry, Nuovo Cimento A 31, 626 (1976).

[36] P. Fayet and J. Iliopoulos, Spontaneously broken supergauge symmetries and Goldstone spinors, Phys. Lett. 51B, 461 (1974).

[37] E. R. Bezerra de Mello, Classical vortex solutions in threedimensional supersymmetric Abelian Higgs model, Mod. Phys. Lett. A 05, 581 (1990).

[38] A. A. Penin, V. A. Rubakov, P. G. Tinyakov, and S. V. Troitsky, What becomes of vortices in theories with flat directions, Phys. Lett. B 389, 13 (1996).

[39] S. C. Davis, A.-C. Davis, and M. Trodden, $N=1$ supersymmetric cosmic strings, Phys. Lett. B 405, 257 (1997).

[40] G. Dvali, R. Kallosh, and A. Van Proeyen, D term strings, J. High Energy Phys. 01 (2004) 035.

[41] E. J. Copeland, R. C. Myers, and J. Polchinski, Cosmic F and D strings, J. High Energy Phys. 06 (2004) 013.

[42] G. Dvali and A. Vilenkin, Formation and evolution of cosmic D strings, J. Cosmol. Astropart. Phys. 03 (2004) 010 .

[43] W. Siegel, Gauge spinor superfield as a scalar multiplet, Phys. Lett. 85B, 333 (1979).

[44] S. J. Gates, Jr., Super p-form gauge superfields, Nucl. Phys. B184, 381 (1981).

[45] U. Lindstrom and M. Rocek, Scalar tensor duality and $N=1, N=2$ nonlinear sigma models, Nucl. Phys. B222, 285 (1983).

[46] S. J. Gates, M. T. Grisaru, M. Rocek, and W. Siegel, Superspace or one thousand and one lessons in supersymmetry, Front. Phys. 58, 1 (1983).

[47] R. D'Auria and S. Ferrara, Dyonic masses from conformal field strengths in D even dimensions, Phys. Lett. B 606, 211 (2005).

[48] J. Louis and W. Schulgin, Massive tensor multiplets in $N=1$ supersymmetry, Fortschr. Phys. 53, 235 (2005).

[49] S. M. Kuzenko, On massive tensor multiplets, J. High Energy Phys. 01 (2005) 041.

[50] J. Louis and J. Swiebodzinski, Couplings of $N=1$ chiral spinor multiplets, Eur. Phys. J. C 51, 731 (2007).

[51] J. Wess and J. Bagger, Supersymmetry and Supergravity (Princeton University, Princeton, NJ, 1992).

[52] A. Gorsky and M. A. Shifman, More on the tensorial central charges in $N=1$ supersymmetric gauge theories (BPS wall junctions and strings), Phys. Rev. D 61, 085001 (2000).

[53] A. Gorsky, M. Shifman, and A. Yung, $N=1$ supersymmetric quantum chromodynamics: How confined nonAbelian monopoles emerge from quark condensation, Phys. Rev. D 75, 065032 (2007).

[54] M. Shifman and A. Yung, Supersymmetric solitons and how they help us understand non-Abelian gauge theories, Rev. Mod. Phys. 79, 1139 (2007).

[55] T. Fujimori, M. Nitta, K. Ohashi, Y. Yamada, and R. Yokokura, Ghost-free vector superfield actions in supersymmetric higher-derivative theories, J. High Energy Phys. 09 (2017) 143.
[56] I. L. Buchbinder, S. Kuzenko, and Z. Yarevskaya, Supersymmetric effective potential: Superfield approach, Nucl. Phys. B411, 665 (1994).

[57] I. L. Buchbinder, S. M. Kuzenko, and A. Yu. Petrov, Superfield chiral effective potential, Phys. Lett. B 321, 372 (1994).

[58] J. Khoury, J.-L. Lehners, and B. Ovrut, Supersymmetric $P(X, \phi)$ and the ghost condensate, Phys. Rev. D 83, 125031 (2011).

[59] M. Koehn, J.-L. Lehners, and B. A. Ovrut, Higher-derivative Chiral superfield actions coupled to $N=1$ supergravity, Phys. Rev. D 86, 085019 (2012).

[60] M. Koehn, J.-L. Lehners, and B. Ovrut, Ghost condensate in $N=1$ supergravity, Phys. Rev. D 87, 065022 (2013).

[61] C. Adam, J. M. Queiruga, J. Sanchez-Guillen, and A. Wereszczynski, Extended supersymmetry and BPS solutions in baby Skyrme models, J. High Energy Phys. 05 (2013) 108.

[62] M. Nitta and S. Sasaki, BPS states in supersymmetric Chiral models with higher derivative terms, Phys. Rev. D 90, 105001 (2014).

[63] M. Nitta and S. Sasaki, Higher derivative corrections to manifestly supersymmetric nonlinear realizations, Phys. Rev. D 90, 105002 (2014).

[64] M. Nitta and S. Sasaki, Classifying BPS states in supersymmetric gauge theories coupled to higher derivative Chiral models, Phys. Rev. D 91, 125025 (2015).

[65] M. Nitta and R. Yokokura, Higher derivative three-form gauge theories and their supersymmetric extension, J. High Energy Phys. 10 (2018) 146.

[66] M. Nitta and R. Yokokura, Topological couplings in higher derivative extensions of supersymmetric three-form gauge theories, J. High Energy Phys. 05 (2019) 102.

[67] E. Cremmer, S. Ferrara, L. Girardello, and A. Van Proeyen, Yang-Mills theories with local supersymmetry: Lagrangian, transformation laws and SuperHiggs effect, Nucl. Phys. B212, 413 (1983).

[68] T. Kugo and S. Uehara, Conformal and Poincare tensor Calculi in $N=1$ supergravity, Nucl. Phys. B226, 49 (1983).

[69] T. Kugo and S. Uehara, Improved superconformal gauge conditions in the $N=1$ supergravity Yang-Mills matter system, Nucl. Phys. B222, 125 (1983).

[70] T. Kugo and S. Uehara, $N=1$ superconformal tensor calculus: Multiplets with external Lorentz indices and spinor derivative operators, Prog. Theor. Phys. 73, 235 (1985).

[71] D. Butter, $N=1$ Conformal superspace in four dimensions, Ann. Phys. (Amsterdam) 325, 1026 (2010).

[72] T. Kugo, R. Yokokura, and K. Yoshioka, Component versus superspace approaches to $D=4, N=1$ conformal supergravity, Prog. Theor. Exp. Phys. 2016, 073 B07 (2016).

[73] T. Kugo, R. Yokokura, and K. Yoshioka, Superspace gauge fixing in Yang-Mills matter-coupled conformal supergravity, Prog. Theor. Exp. Phys. 2016, 093 B03 (2016).

[74] S. Aoki, T. Higaki, Y. Yamada, and R. Yokokura, Abelian tensor hierarchy in $4 \mathrm{D} \mathcal{N}=1$ conformal supergravity, J. High Energy Phys. 09 (2016) 148.

[75] R. Yokokura, Abelian tensor hierarchy and Chern-Simons actions in $4 \mathrm{D} \mathcal{N}=1$ conformal supergravity, J. High Energy Phys. 12 (2016) 092. 
[76] A. Hanany and D. Tong, Vortices, instantons and branes, J. High Energy Phys. 07 (2003) 037.

[77] R. Auzzi, S. Bolognesi, J. Evslin, K. Konishi, and A. Yung, NonAbelian superconductors: vortices and confinement in $N=2$ SQCD, Nucl. Phys. B673, 187 (2003).

[78] M. Eto, Y. Isozumi, M. Nitta, K. Ohashi, and N. Sakai, Instantons in the Higgs phase, Phys. Rev. D 72, 025011 (2005).

[79] M. Eto, Y. Isozumi, M. Nitta, K. Ohashi, and N. Sakai, Moduli Space of Non-Abelian Vortices, Phys. Rev. Lett. 96, 161601 (2006).

[80] A. Gorsky, M. Shifman, and A. Yung, Non-Abelian meissner effect in Yang-Mills theories at weak coupling, Phys. Rev. D 71, 045010 (2005).

[81] M. Eto, K. Konishi, G. Marmorini, M. Nitta, K. Ohashi, W. Vinci, and N. Yokoi, Non-Abelian vortices of higher winding numbers, Phys. Rev. D 74, 065021 (2006).

[82] M. Eto, K. Hashimoto, G. Marmorini, M. Nitta, K. Ohashi, and W. Vinci, Universal Reconnection of NonAbelian Cosmic Strings, Phys. Rev. Lett. 98, 091602 (2007).

[83] D. Tong, TASI lectures on solitons: Instantons, monopoles, vortices and kinks, arXiv:hep-th/0509216.

[84] M. Eto, Y. Isozumi, M. Nitta, K. Ohashi, and N. Sakai, Solitons in the Higgs phase: The Moduli matrix approach, J. Phys. A 39, R315 (2006).

[85] Y. Hirono and Y. Tanizaki, Quark-Hadron Continuity beyond Ginzburg-Landau Paradigm, Phys. Rev. Lett. 122, 212001 (2019).

[86] T. E. Clark, C. H. Lee, and S. T. Love, Supersymmetric tensor gauge theories, Mod. Phys. Lett. A 04, 1343 (1989).
[87] K. Furuta, T. Inami, H. Nakajima, and M. Nitta, Supersymmetric extension of nonAbelian scalar tensor duality, Prog. Theor. Phys. 106, 851 (2001).

[88] C. Chatterjee and M. Nitta, BPS alice strings, J. High Energy Phys. 09 (2017) 046.

[89] C. Chatterjee and M. Nitta, The effective action of a BPS Alice string, Eur. Phys. J. C 77, 809 (2017).

[90] J. P. Gauntlett, R. Portugues, D. Tong, and P. K. Townsend, D-brane solitons in supersymmetric sigma models, Phys. Rev. D 63, 085002 (2001).

[91] M. Shifman and A. Yung, Domain walls and flux tubes in $N=2$ SQCD: D-brane prototypes, Phys. Rev. D 67, 125007 (2003).

[92] Y. Isozumi, M. Nitta, K. Ohashi, and N. Sakai, All exact solutions of a 1/4 Bogomol'nyi-Prasad-Sommerfield equation, Phys. Rev. D 71, 065018 (2005).

[93] M. Shifman and A. Yung, NonAbelian string junctions as confined monopoles, Phys. Rev. D 70, 045004 (2004).

[94] A. Hanany and D. Tong, Vortex strings and four-dimensional gauge dynamics, J. High Energy Phys. 04 (2004) 066.

[95] M. Nitta and W. Vinci, Non-Abelian monopoles in the Higgs phase, Nucl. Phys. B848, 121 (2011).

[96] M. Naganuma, M. Nitta, and N. Sakai, BPS lumps and their intersections in $N=2$ SUSY nonlinear sigma models, Gravitation Cosmol. 8, 129 (2002).

[97] M. Eto, Y. Isozumi, M. Nitta, and K. Ohashi, 1/2, 1/4 and 1/8 BPS equations in SUSY Yang-Mills-Higgs systems: Field theoretical brane configurations, Nucl. Phys. B752, 140 (2006).

[98] C. Chatterjee and K. Konishi, Monopole-vortex complex at large distances and nonAbelian duality, J. High Energy Phys. 09 (2014) 039. 